\title{
On Limitations of Friendly Jamming for Confidentiality
}

\author{
Nils Ole Tippenhauer, Luka Malisa, Aanjhan Ranganathan, Srdjan Capkun \\ Institute of Information Security \\ ETH Zurich \\ Zurich, Switzerland \\ \{tinils,malisal,raanjhan,capkuns\}@inf.ethz.ch
}

\begin{abstract}
Wireless communication provides unique security challenges, but also enables novel ways to defend against attacks. In the past few years, a number of works discussed the use of friendly jamming to protect the confidentiality of the communicated data as well as to enable message authentication and access control. In this work, we analytically and experimentally evaluate the confidentiality that can be achieved by the use of friendly jamming, given an attacker with multiple receiving antennas. We construct a MIMO-based attack that allows the attacker to recover data protected by friendly jamming and refine the conditions for which this attack is most effective. Our attack shows that friendly jamming cannot provide strong confidentiality guarantees in all settings. We further test our attack in a setting where friendly jamming is used to protect the communication to medical implants.
\end{abstract}

\section{INTRODUCTION}

The shared nature of the wireless communication channel poses numerous security challenges, from eavesdropping by a nearby attacker to selective interruption of communication by jamming. However, the physical layer of wireless communications also enables novel ways to defend against attacks. For example, wireless transceivers can collaborate in order to prevent an attacker from interfering with, as well as eavesdropping on, their communication. Recently, a number of schemes were proposed in this space [1]-[7]; these schemes use the idea of friendly jamming - intentional signal interference by collaborating and coordinated devices.

Friendly jamming is typically used to achieve the following goals: (i) to prevent an attacker from communicating with a protected device, and (ii) to prevent the attacker from eavesdropping on messages sent by protected devices. The first goal is related to access control, authentication and intrusion detection, and is typically achieved by a friendly jammer who jams all traffic to or from a protected device [8]-[10]. The second goal is confidentiality and is achieved in this setting by exposing the attacker to friendly jamming such that the attacker's channel, unlike the channel of the protected receiver, is degraded to such an extent that successful decoding of messages becomes infeasible.

We focus on confidentiality provided by friendly jamming schemes. Although it might seem that the use of jamming for confidentiality is unnecessary because confidentiality can be simply achieved by encryption, there are numerous scenarios where key distribution, performance, mismanagement, or legacy issues prevent the establishment or use of shared keys. One example is the case of medical implants, where access to the implant data is safety-critical and must be granted to medical professionals in all circumstances, even in foreign domains to which appropriate credentials or keys cannot be distributed [11], [12]. Another example is a corporate setting where friendly jamming can be used as a second layer of protection against unintentional information leakage, mitigating the risk that misconfigured or legacy systems do not use appropriate encryption [13]. Furthermore, jamming can be used to protect initial key establishment, e.g. in sensor networks [14].

Friendly jamming schemes that aim to achieve confidentiality rely on the assumption that it will be hard for the attacker to extract the message, as the jamming signal from the friendly jammer and the signal carrying the confidential message superimpose at the attacker's antenna(s). The signal at the attacker's antennas and thus his ability to extract the message depends on many factors: the jamming and message signals, the mutual distances between the jammer, message source and the attacker, their locations as well as the environment itself. Some related work on friendly jamming assume scenarios where the attacker has limited capabilities in terms of the number or directionality of his antennas and antenna placement [1], [2], [15]. Other related works such as [11], [16] do not assume an attacker that is restricted in terms of the number of antennas but instead argue that one can defend against a MIMO eavesdropper or an eavesdropper with a directional antenna by ensuring that the friendly jammer is located significantly less than half a wavelength from the data source. These works also show that if the attacker is equipped with a single antenna, proximity alone is not sufficient for confidentiality, but that the ratio between the jammer and data source power is equally a critical parameter. These arguments are based on channel models and signal processing techniques commonly used in wireless communication research. However, the effectiveness of friendly jamming has so far not been studied in scenarios in which the attacker is equipped with multiple antennas.

In this work we analyze friendly jamming in scenarios where the friendly jammer and the message source are physically very close (closer than half of the signal's carrier 


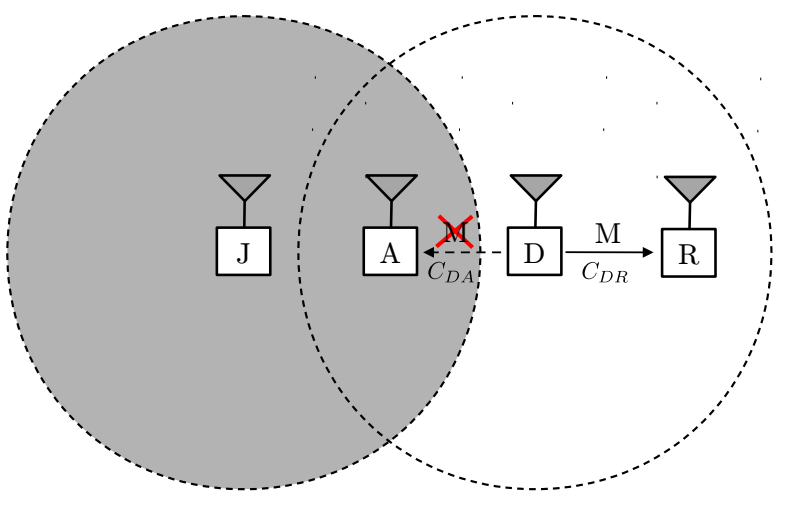

(a)

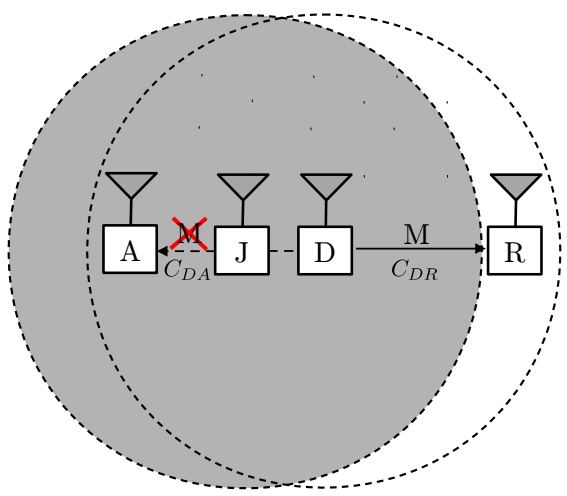

(b)

Figure 1. Two common friendly jamming scenarios. In both, the attacker $A$ is jammed by $J$ and is unable to eavesdrop on a message $M$ transmitted by the transmitter $D$. The legitimate receiver $R$ can still receive the message. We differentiate between the scenario in which the data source ( $D$ ) and friendly jammer $(J)$ are far (a) and when they are close (b).

wavelength), given an attacker with two receiving antennas. We introduce a new type of an attack, based on MIMO, that allows the attacker to recover confidential messages from distances sufficient for a practical attack. We demonstrate our attack both analytically and experimentally.

In our analytical evaluation, based on a Line-of-Sight (LoS) model, we show that the attacker can recover the confidential message even when the friendly jammer and the data source are few centimetres apart and the attacker is several meters away.

Our experiments were done in the $402-405 \mathrm{MHz}$ MICS band. In our LoS experiments, the friendly jammer and the data source were placed $15-30 \mathrm{~cm}$ apart (less than half a wavelength for the MICS band). In this setting, we demonstrate the successful recovery of confidential messages by an attacker from up to $3 \mathrm{~m}$ away, and in some cases from further distances. In our NLoS measurements, we placed the data source (a USRP) behind a layer of bacon and ground beef, simulating a medical implant in human flesh and we positioned the friendly jammer within $5-7 \mathrm{~cm}$ of the data source. In this setting we were able to recover confidential messages from distances of up to $2 \mathrm{~m}$ with success rates depending on environmental conditions. The above distances between the jammer, data source and the attacker correspond to many practical settings. Our results also raise security concerns with the use of friendly jamming for the protection of confidentiality of messages transmitted by medical implants. ${ }^{1}$

\footnotetext{
${ }^{1}$ In our experimental results we shape the jamming signal power profile for the FSK modulated data that our data source uses. The works in [11], [16] generate friendly jamming signal by combining multiple random white Gaussian signals and by matching the shape of their power profile to that of the implant. In this work, we do not restrict the type of jamming signal that the friendly jammer generates as our attack helps in eliminating the jamming signal regardless of its type.
}

In this work we consider a relatively strong attacker, who can place his antennas at chosen locations in the vicinity of the victim's system. Nevertheless, we believe that in a number of scenarios, especially those where the message source and the jammer are static (e.g., a patient lies stationary during medical examination or a computer that is used in an office), this attacker model is practical. In the case of a mobile victim, our attack can still be used to intermittently recover parts of transmitted messages, violating the confidentiality provided by friendly jamming schemes. Note that since the transmitted messages are not protected by any form of encryption, but that their confidentiality is typically solely protected by friendly jamming, the attacker may be able to recover full messages even if he is able to receive only individual message fragments.

Through our analysis and experiments, we demonstrate a fundamental limitation of friendly jamming schemes when they are used for confidentiality and we refine the conditions under which such schemes can be used. Our results however, do not pertain only to friendly jamming, but can also be seen as a further refinement of attacker models used in the analysis of physical-layer security schemes - they highlight again the importance of precise modeling of attacker's capabilities.

The structure of the paper is as follows. We give background information on friendly jamming schemes in Section II. We then analyze the underlying model and assumptions in detail in Section III and show analytically under which circumstances the attacker can eliminate the jamming signal. We validate our findings experimentally in Section IV and show that a practical attacker can remove the jamming signals and recover the data. In Section V, we discuss the impact of our findings and possible countermeasures. We conclude the paper in Section VI. 


\section{BACKGROUND}

In the context of confidentiality, friendly jamming is used in a scenario in which devices rely on a friendly jammer to prevent the attacker from overhearing the messages that they exchange. A typical friendly jamming scenario consists of four parties: a transmitter, receiver, attacker and a jammer. The jammer's goal is to sufficiently degrade the channel between the transmitter and the attacker such that the attacker cannot decode the messages coming from the transmitter. However, the jammer must not prevent the receiver from decoding the messages.

We differentiate between two types of friendly jammer systems:

- Remote jammer systems. In these systems, the distance between the transmitter and the jammer is much larger than half a carrier wavelength. Here, a common security assumption is that the attacker is equipped with a single omni-directional antenna and thus cannot separate the confidential message from the jamming signal. However, in a number of scenarios, an attacker can still try to use antenna directionality to his advantage and extract the exchanged message. A remote jammer system is illustrated in Figure 1(a).

- Nearby jammer systems. In these systems, the jammer and the transmitter are less than half a carrier wavelength apart. The main security assumption here is that, in these systems, the jamming and data signal envelopes are highly correlated ( [17] $\S$ 13.2.1) and are thus assumed to be inseparable. We later show that this assumption does not always hold. A nearby jammer system is illustrated in Figure 1(b).

\section{A. Friendly Jamming Schemes}

Secrecy capacity [18] is a key notion in the informationtheoretic aspects of wireless security. It is defined as the difference between the capacity of the intended communication channel and the capacity of the eavesdropper channel. Prior work discusses a number of ways of quantifying and increasing the secrecy capacity bounds of various types of wireless channels. Proposed systems rely on one or more relay nodes [1]-[4], multi-antenna transmitters [19] or friendly jammers [5], [6] to increase secrecy capacity by enhancing the receiver's and degrading the attacker's channel. Proposed systems considered a single [6] as well as multiple friendly jammers [7]. All the schemes primarily considered only remote jamming.

A common attacker model used in the analysis of these schemes was based on a single, passive eavesdropper [4][6], [20] and if resilience against multiple eavesdroppers was considered, eavesdropper collusion was not made a part of the model [1], [19].

Goel and Negi [21] consider the case of transmitter, receiver and attacker all having multiple antennas. However,

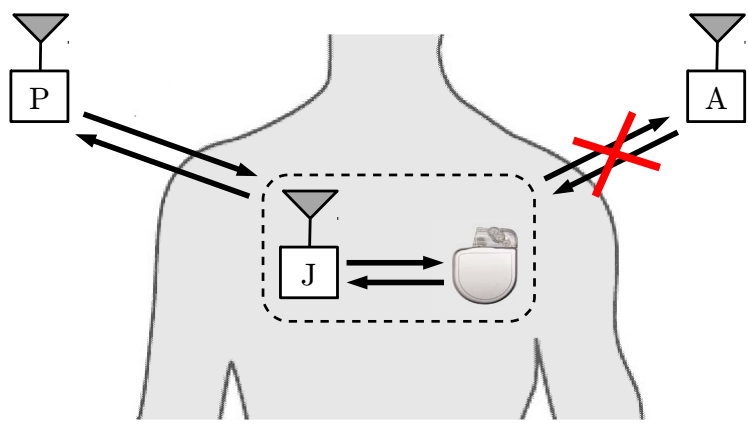

Figure 2. The scheme proposed in [11]: To secure communication to the implant, the patient wears an IMD shield. The shield $(J)$ serves as a friendly jammer and prevents all direct communication to an IMD and makes sure that unauthorized devices $(A)$ cannot decode messages transmitted by the IMD. A programming device $(P)$ which wants to communicate with the IMD has to first establish a secure channel with the shield.

the total number of attacker's antennas in their analysis is less than or equal to the number of transmitting antennas.

Pinto et al. [22] consider a more powerful attacker. They analyze the secrecy capacity in the presence of colluding, spatially-separated eavesdroppers. Within their model, the eavesdroppers are allowed to share and combine information to improve their chances of successfully decoding a message. The authors conclude that an increasing number of colluding eavesdroppers drastically reduces the achievable secrecy capacity.

Kuo et al. consider the problem of secure initialization of nodes in sensor networks [14]. The proposed scheme employs a Faraday cage in order to significantly attenuate signals which carry secret keying information. A friendly jammer is placed outside of the Faraday cage to further interfere with any potential eavesdroppers.

In the following, we describe a scheme that considered the use of nearby jamming.

Gollakota et al. propose the use of nearby jamming to ensure confidentiality and access control to implantable medical devices (IMDs) [11].

In this scheme, a friendly jammer aims to protect an "insecure" IMD, i.e., an IMD that does not implement any security mechanisms. Without this protection, an attacker could freely communicate with, as well as eavesdrop on, data transmitted by the IMD. This would pose both safety risks (malicious reconfiguration of the device) and privacy concerns (leakage of private information). To protect against these attacks, the user wears an additional device on a necklace-the shield. The shield acts as a gateway for external devices who want to communicate with the IMD - it will prevent any direct external communication to the IMD by jamming unauthorized queries of the IMD. In addition, the shield will jam any transmissions from the IMD, preventing anyone from eavesdropping on the message content. The shield itself can cancel out his own jamming signal and can thus correctly demodulate the data sent by the IMD. 
Consequently, anyone who wishes to communicate with the IMD must first contact the shield, who then forwards the communication to the IMD. Because the shield is an external device, unlike an IMD, it can easily establish a secure channel to any legitimate reader.

For an attacker with a single antenna, the scheme argues that given close proximity of the jammer to the data source, sufficient jamming power, and a well-formed jamming signal, an attacker cannot recover the data signal. To defend against an attacker with multiple antennas, the work assumes that the respective channels of the IMD and of the shield to the attacker cannot be separated with directional antennas or techniques such as MIMO if the IMD and the shield are significantly less than half of the communication signal wavelength apart. As reasoning for this, it refers to Chapter 1 in [23] and Chapter 7 in [24] — these references, however, do not explore the use of MIMO-like techniques in adversarial settings. In the example of medical implants, this work considers a distance of few centimeters, without giving a precise threshold. In terms of the attacker, the only other restriction that is imposed is that the attacker must be located further away from the jammer than the implant (e.g., at distances $20 \mathrm{~cm}$ and greater). The attacker is not restricted in terms of the number, location, or directionality of the antennas that he uses. Despite considering a strong attacker, the work focuses the evaluation of the proposed scheme on an attacker with a single antenna.

\section{JAmming Mitigation Using Channel RESOLUTION}

Friendly jamming can only achieve data confidentiality if the attacker cannot recover the data signal. Remote jammer schemes, where the friendly jammer is far $(>\lambda / 2)$ from the transmitting device, were studied in prior work and their limitations in terms of attacker placement as well as number and directionality of his antennas are well known. Nearby jammer schemes promised to significantly raise the security guarantees, based on the assumption that the friendly jammer and the transmitting device are collocated. They pose no special restrictions on the attacker, except that he is not too close to the jammer or the transmitter. We focus on nearby jammer schemes and show that their security guarantees are weaker than it was so far believed.

The security of friendly jamming schemes is typically argued by considering signal strengths, non-separability of channels using MIMO techniques, and the information rate of the combined data/jamming signal. Here, we investigate channel non-separability arguments in more detail and analyze whether it is possible for the attacker to use techniques similar to MIMO in order to separate the jamming and data signal even if the jammer and transmitter are close.

We begin with a brief example of the attack to give an intuition, and then derive why the attacker, in our setting, is able to separate the data signal from the jamming signal.

\section{A. Example of our Attack}

Consider the following scenario shown in Figure $3 \mathrm{~b}$. The attacker is equipped with two antennas ( $A$ and $B$ ) which are equidistant from the jamming source $(\overline{A J}=\overline{B J})$, but not equidistant from the data source $(\overline{A D} \neq \overline{B D})$. Both antennas receive a composite signal, consisting of a data component as well as a jamming component. As a result, the received signal at $A$ and $B$ have each a different relative phase offsets between the data and signal component.

Because both antennas of the attacker are equidistant to the jammer, the signal received by both antennas will contain the jamming signal with same phase. The data component is received with some phase offset $\delta$ depending on the geometric setting. Lets assume that in this example, the phase shift is equal to half a wavelength, i.e., $\delta=\pi$ (in radians). In Figure $3 \mathrm{a}$ we depict the two received signals as well as their individual components. By subtracting the received signals, the attacker can remove the jamming component and obtain an amplified data component. Since the jamming components have the same amplitude and phase, it is easy to see that they are eliminated when the signals are subtracted. Differently, when two $\lambda / 2$-shifted data components are subtracted, they constructively interfere, resulting in an amplified data signal.

From this example, it follows that there are indeed constellations in which the attacker can recover the data signal. We now investigate these conditions in more detail. We first briefly review a standard MIMO line-of-sight (LOS) channel model which states that the channel should not be resolvable and signals should not be separable. We show why this channel model does not exactly capture our use case here-it requires a certain minimal distance between sender and receiver. We then compute the approximation error of this standard model for the nearby jammer case. Finally, we identify a way to separate the data and jamming channel leveraging the observation on the approximation error and show, for selected settings, resulting bit error rates of the attacker when he recovers the data signal.

\section{B. MIMO Basics}

In this context, multiple-input and multiple-output (MIMO) denotes a setting in which a transmitter uses multiple antennas to transmit a wireless message to a receiver with multiple antennas. Among other aspects, the channel diversity between the transmitting and receiving antennas enables the receiver to improve the received signal quality in presence of fading channels and can also be used to improve spectral efficiency. For example, multiple antennas can provide a simple power gain when aligned correctly. Multiple antennas can also help to mitigate random effects of channel fading. A normal MIMO setting assumes that sender and receiver are collaborating to improve their communication. In our setting, we are investigating whether the attacker can recover signals sent by the transmitter in the presence of 


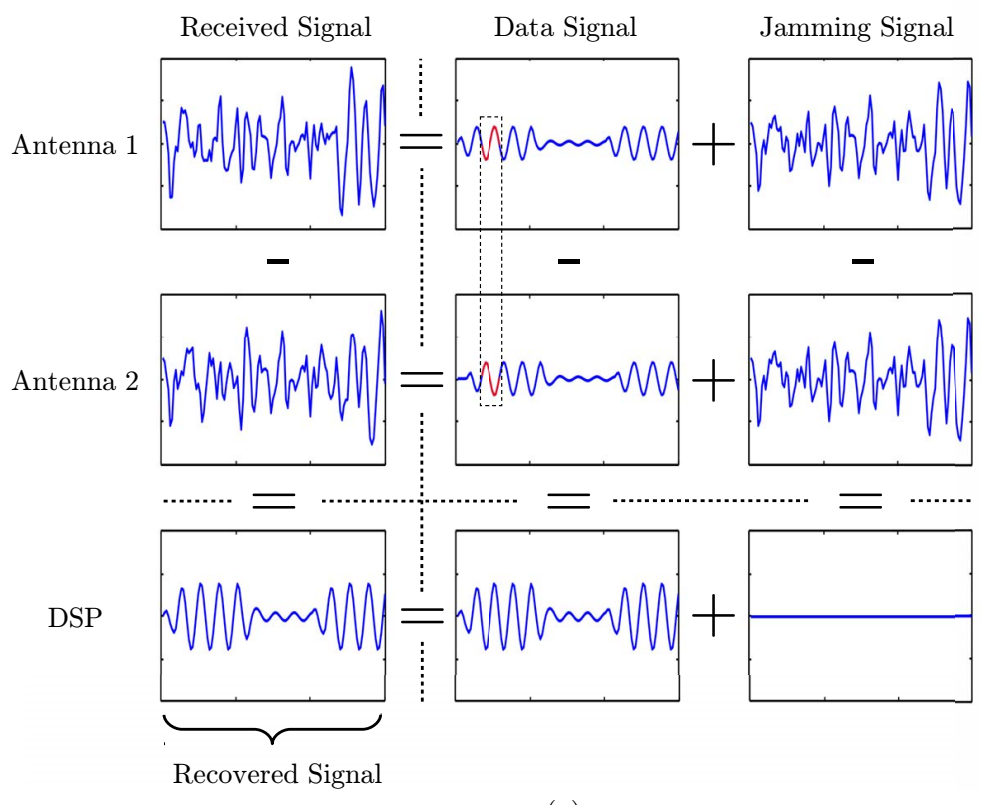

(a)

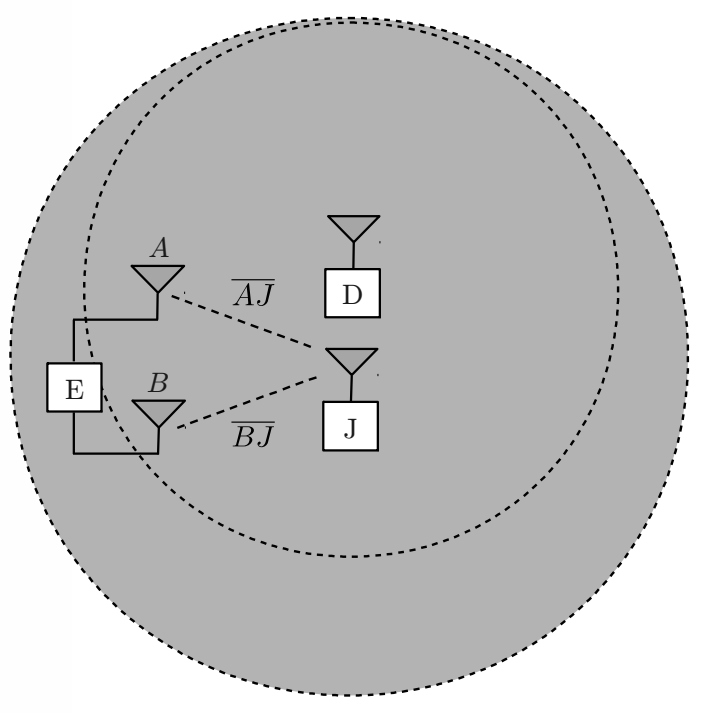

(b)

Figure 3. A simplified scenario which illustrates our attack. Both the data source $D$ and attacker $E$ are within the jammed area. In this example, the attacker is equipped with two antennas $(A$ and $B)$ which are equidistant from the jamming source, i.e., $\overline{A J}=\overline{B J}$. The antennas are not equidistant from the data source.

a jammer, without the collaboration of neither jammer nor transmitter. For a thorough introduction into MIMO, we refer to [24].

\section{Line-of-Sight MIMO Model}

For our discussion, we assume that the attacker has two omni directional antennas $(A, B)$ which he can place at any distance to the jammer $J$ that is greater than the distance between the jammer $J$ and the data source $D$ antennas. We further consider a case in which the transmitting antennas $(D$ and $J$ ) are placed less than $\lambda / 2$ apart from each other.

A standard way to model wireless transmissions in our setting is the following description:

$$
y=H x+w
$$

Applied to our use-case, $y=\left[\begin{array}{ll}s_{A} & s_{B}\end{array}\right]^{T}$ is the column vector of signals received by the attacker, $H$ is a channel matrix, $x=\left[\begin{array}{ll}s_{d} & s_{j}\end{array}\right]^{T}$ is the vector of source signals and $w$ is additional random channel noise. For the following discussion, we omit the noise to simplify the equations.

The channel matrix $H$ depends on the chosen channel model. Here, we approximate the channel as several lineof-sight paths. For this setting, a standard channel model can be found in $\S 7.2 .3$ in [24], on which we will base our discussion. We call the resulting channel matrix $H^{m}$ to differentiate from a second model we will introduce later.

The channel model $H^{m}$ assumes that all transmitted signals experience the same channel conditions and arrive

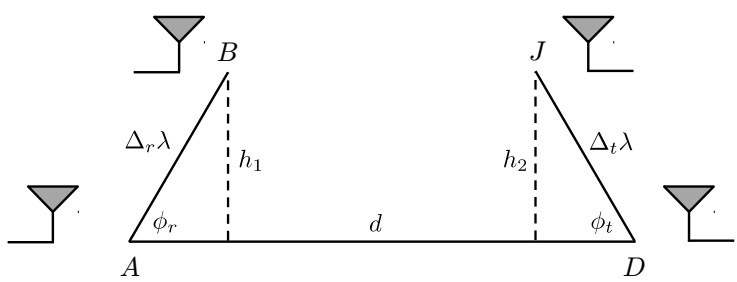

Figure 4. The geometric model for the IMD shield use case.

only with small phase offsets approximated based on the geometric setup. To simplify the computations, the model assumes that all signals are received along the same direction between $A$ and $D$ (shown in Figure 4). The relative position of the other receiving antenna with regards to $A$ is expressed as distance $\Delta_{r} \lambda$ and the angle $\phi_{r}$, the same for the transmitting array with $\phi_{t}$ and $\Delta_{t} \lambda$, respectively. $\Delta_{t}$ and $\Delta_{r}$ are the length of the transmit and receive arrays, normalized to the wavelength $\lambda$. To simplify the formulas, we define $\Omega:=\cos \phi$. Based on these variables, the model presents $H^{m}$ as:

$$
H^{m}=2 a \exp \left(-\frac{j 2 \pi d}{\lambda}\right) e_{r}\left(\Omega_{r}\right) e_{t}\left(\Omega_{t}\right) *
$$

with

$$
e_{r}(\Omega):=\frac{1}{\sqrt{2}}\left[\begin{array}{c}
1 \\
\exp \left(-j 2 \pi \Delta_{r} \Omega\right)
\end{array}\right]
$$




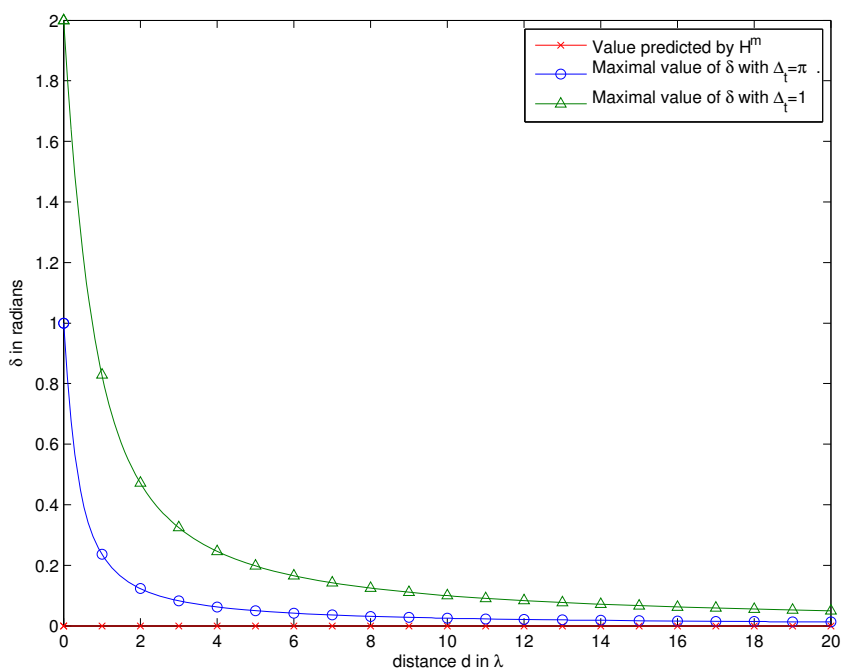

Figure 5. Approximation error for $\delta$ in the rectangular formation setting with $\Delta_{r}=\Delta_{t}=0.5$.

and

$$
e_{t}(\Omega) *:=\frac{1}{\sqrt{2}}\left[\begin{array}{cc}
1 & \exp \left(-j 2 \pi \Delta_{t} \Omega\right)
\end{array}\right]
$$

and an attenuation factor $a$.

It can be seen that $H^{m}$ has rank 1 and thus $y$ must also have rank at most 1 . Indeed, although $y$ has a dimension equal to the number of antennas of the receiver, all antennas essentially receive a linear combination of the same signals if the rank of $y$ is 1 . It follows that the sources cannot be separated based on these linear combinations alone.

In this way, the channel model $H^{m}$ states that the attacker cannot eliminate a second nearby signal source such as a friendly jammer.

\section{Analysis of Approximation Error}

In $\S 7.2 .3$ in [24], an assumption for the MIMO model we used was:

"Assuming again that the antenna array sizes are much smaller than the distance between the transmitter and the receiver $[\ldots]$ "

We now examine the approximation error of this LOS MIMO model for our use case (in which the antenna array sizes is not "much smaller" than the distance) and how this approximation error influences the security of the proposed friendly jamming scheme.

To summarize the parameters for the model, in our use case we have two antenna arrays (RX/TX), with two antennas each. The two antennas of the transmitting array are set up such that $\Delta_{t} \leq 0.5$. The distance $d$ between the transmitting and receiving array is between 1 and 10 wavelengths, thus violating the model assumption regarding distance. In this setting, we are in particular interested in the phase offset between the two transmitted signals $s_{d}, s_{n}$ at the two receiving antennas.

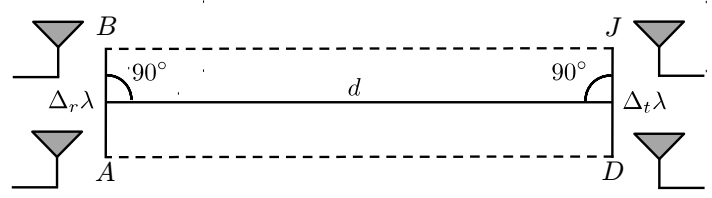

Figure 6. A simple rectangular model with $\Delta_{r} \lambda=\Delta_{t} \lambda$.

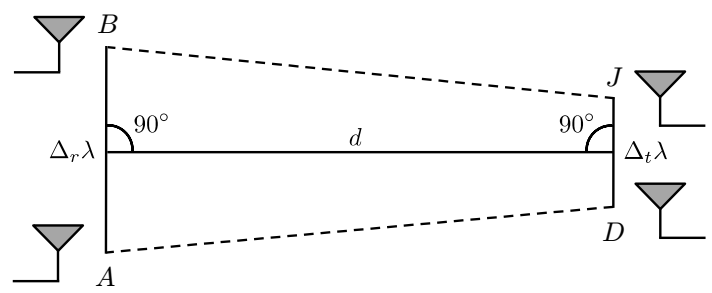

Figure 7. Geometry of the simulations: all 4 antennas form an isosceles trapezoid. The simulations are run for varying $d$ and $\overline{A B}$

For simplicity, we will start with showing the approximation error for a rectangular formation (Figure 6) with distance $d$ between the RX/TX arrays and each arrays with two antennas $\Delta_{r}=\Delta_{t}$ apart (both distances measured in carrier wavelengths). For this, we insert $\phi_{t}=\phi_{r}=90^{\circ}$ (via $\Omega_{r}$ and $\Omega_{t}$ ) into Equation 2 and Equation 3, which allows us to rewrite Equation 1 as the following channel matrix, which we will denote as $H^{m}$ :

$$
H^{m}=a\left[\begin{array}{ll}
e^{-j 2 \pi d / \lambda} & e^{-j 2 \pi d / \lambda} \\
e^{-j 2 \pi d / \lambda} & e^{-j 2 \pi d / \lambda}
\end{array}\right]
$$

Based on the geometry of the formation, we now also compute the correct phase shifts, and derive our correct channel matrix, which we call $H^{g}$ ), as

$$
H^{g}=a\left[\begin{array}{cc}
e^{-j 2 \pi d / \lambda} & e^{-j 2 \pi \sqrt{d^{2}+\Delta_{t}^{2}} / \lambda} \\
e^{-j 2 \pi \sqrt{d^{2}+\Delta_{t}^{2}} / \lambda} & e^{-j 2 \pi d / \lambda}
\end{array}\right]
$$

As $H^{g}$ is clearly different to $H^{m}$ (e.g. $H^{g}$ has rank 2 for $\Delta_{t}>0$ and $a \neq 0$ ), the simplified model $H^{m}$ has to have some approximation error. In particular, we are interested in the difference of phase offsets of the data and jamming signal, i.e. $\delta=\angle h_{11}-\angle h_{12}-\angle h_{21}+\angle h_{22}$, where $\angle h_{i j}$ is the phase of the $i$ th row and $j$ th column entry of a channel matrix $H$. For the model from [24], $\delta(d)=0$. In Figure 5, we show the approximation error of the model for small distances $d$, up to 20 times the carrier wavelength. It can be seen that up to a distance of 2.5 wavelengths, $\delta$ can reach 0.1 and more. As we show later, such values for $\delta$ can allow the attacker to eliminate the noise signal and recover the data signal perfectly. Thus, the simplified approximation in $H^{m}$ should only apply to values of $d$ much larger than $\lambda$, as was the condition stated for the model. 


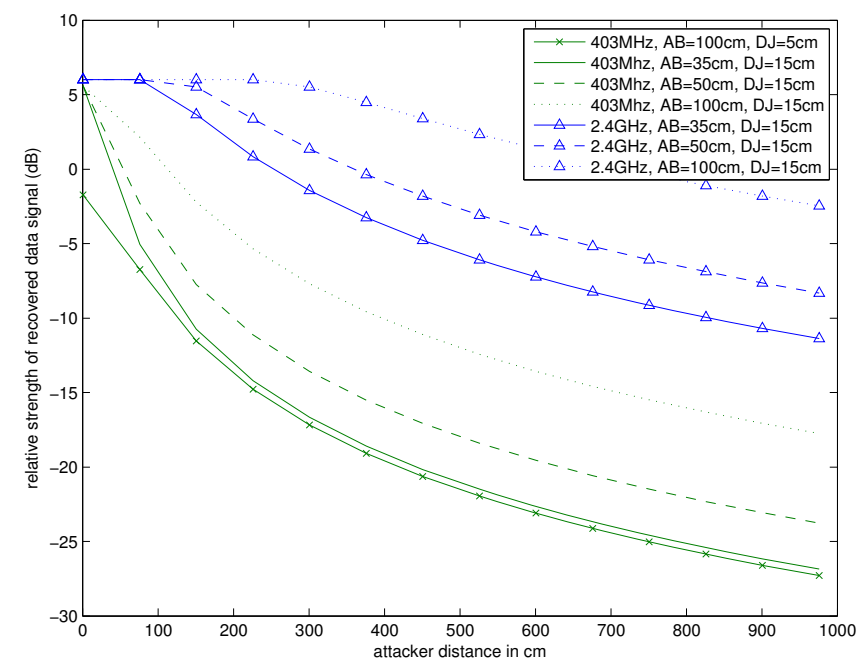

Figure 8. Theoretical limits for data signal amplification with simultaneous jamming signal elimination. In this plot, we assume an isosceles trapezoid formation between the signal sources and the attacker.

\section{E. Description of Our Attack}

Our attack allows the attacker to mitigate the jamming signal and recover the data signal in the presence of friendly jamming. The adversary uses a two-antenna array that he places in the proximity of the transmitting array which consists of a data source $(D)$ and a jammer $(J)$. As we derived earlier, for small distances between the two arrays, the attacker receives the two sets of jamming and data signals with a non-negligible phase offset $\delta$. This $\delta$ allows the attacker to combine the signals received by $A$ and $B$ such that the jamming signal component is annihilated and the data signal recovered.

More precisely, the attacker combines the signals at $A$ and $B$ to recover a signal $\tilde{y}=c \exp \left(-j 2 \pi \Delta_{c}\right) s_{d}$, i.e., a phase-shifted (by $\Delta_{c}$ ) version of the data signal from $D$ with different amplitude $c$. This signal combination can be described by a vector $r$, where

$$
\tilde{y}=r H x
$$

For the example setting before, choosing $r^{g}=\left[\begin{array}{ll}h_{22}^{g} & -h_{12}^{g}\end{array}\right]$ causes the $s_{j}$ component to disappear from $\tilde{y}$. In other words, let $r^{g}$ be

$$
r^{g}=\left[\begin{array}{ll}
e^{-j 2 \pi d} & -e^{-j 2 \pi \sqrt{d^{2}+\Delta_{t}^{2}}}
\end{array}\right]
$$

then consequently

$$
\tilde{y}=\left[\begin{array}{c}
e^{-j 2 \pi d} \\
-e^{-j 2 \pi \sqrt{d^{2}+\Delta_{t}^{2}}}
\end{array}\right]^{T} a\left[\begin{array}{cc}
e^{-j 2 \pi d} & e^{-j 2 \pi \sqrt{d^{2}+\Delta_{t}^{2}}} \\
e^{-j 2 \pi \sqrt{d^{2}+\Delta_{t}^{2}}} & e^{-j 2 \pi d}
\end{array}\right] x
$$

which can be simplified as

$$
\tilde{y}=a \cdot s_{d}\left(e^{-j 4 \pi d}-e^{-j 4 \pi \sqrt{d^{2}+\Delta_{t}^{2}}}\right) .
$$

Thus, using $r^{g}$ we obtain $\tilde{y}$, a phase shifted and amplitude changed version of $s_{d}$, where both amplitude and phase

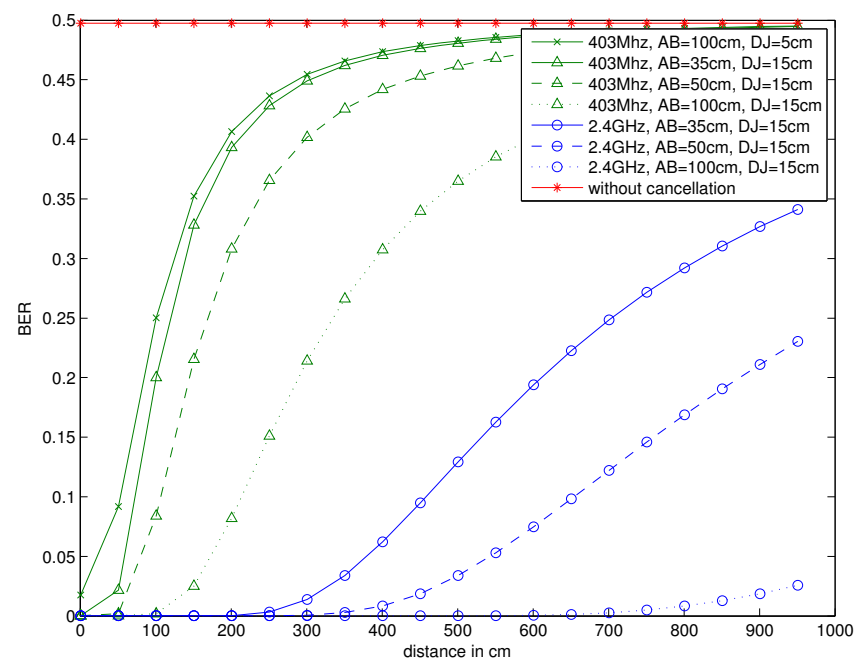

Figure 9. Simulation of expected BER at attacker before and after jamming cancellation. The results are plotted for different carrier frequencies and distances between the attacker's antennas. This simulation assumes a $16 \mathrm{~dB}$ SNR in absence of the jamming signal and considers the $w$ ignored earlier.

depend on the geometry of the setup, in this case $d$ and $\Delta_{t}$. Note that the difference between the phases of both terms here is the $\delta$ discussed earlier. The closer $\delta$ is to $\pm \pi$ (in radians), the higher the amplitude of $s_{d}$ in $\tilde{y}$. The achievable $\delta$ directly depends on $r$, which itself depends on the geometry of the setup.

\section{F. Attack Performance in LOS Setting}

We now perform simulations to evaluate the performance of the attack for different values of $d$ and $\overline{A B}$. Due to the many degrees of freedom for the placement of two antennas of the attacker with respect to $D$ and $J$, we restrict this analysis to a symmetric setting, in which the four antennas form an isosceles trapezoid. While this setting does not cover all possible placements of the attacker's antennas relative to $D$ and $J$, it shows how the attack performs with respect to the distance between $A$ and $B$, and the general distance between attacker and $D$ and $J$.

In the setting used in the simulations (Figure 7), all 4 antennas form an isosceles trapezoid where the TX antennas are $15 \mathrm{~cm}$ apart (unless noted otherwise), and the RX antennas are separated by either $35 \mathrm{~cm}, 50 \mathrm{~cm}$, or $100 \mathrm{~cm}$. We now investigate the performance for different distances $d$ between both antenna arrays. Figure 8 shows the maximal amplitude of the recovered data signal $s_{d}$ in $\tilde{y}$, relative to the amplitude $s_{d}$ would have at $A$ in the absence of $J$. Therefore, a relative amplitude of 2 denotes a perfect recovery of the data signal, in which the data components of both antennas constructively add together and double the amplitude. It can be seen that even for a close spacing of the TX antennas, $s_{d}$ can be recovered from a distance of multiple meters, depending on RX setup, with some attenuation of less than $20 \mathrm{~dB}$. Because of the different wavelength of bands, such 
as the $403 \mathrm{MHz}$ MICS band and the $2.4 \mathrm{GHz}$ ISM band, the values of $\delta$ differ, and therefore the amplitude of the recovered signal differs as well.

We now estimate the expected bit error rate (BER) at the attacker to make the impact of our attack more intuitive. For this, we assume the following: the transmitting and receiving antennas are in the same formation as before (Figure 7), with the data and jamming antenna spaced $15 \mathrm{~cm}$ apart. We further assume that the attacker can receive the data signal with low BER in the absence of the jamming signal, so we assume his signal-to-noise ratio (SNR) to be $16 \mathrm{~dB}$ in the absence of the jamming signal. We now also account for the receiver noise $w$ we ignored earlier, and assume that the noise power at both antennas is the same, but the noise is uncorrelated. Figure 9 shows the impact of the friendly jamming signal transmitted with $20 \mathrm{~dB}$ more than data signal, for three different configurations of attacker's antennas (antennas placed 35,50,100 cm apart). For the case of $\overline{A B}=100 \mathrm{~cm}$, we also show results for data and jamming source $5 \mathrm{~cm}$ apart. For reference, we also again also plot data for the ISM band. We now point to a number of observations that can be made from the figure. First, without jamming cancellation, the BER at the attacker is $50 \%$, as expected. This means that if the attacker does not try to cancel out jamming, he is not able to recover any data from the transmission. Second, if the attacker's antennas are placed $50 \mathrm{~cm}$ apart, the attacker can receive the data signal with a fairly low BER from a distance of up to $1 \mathrm{~m}$ from the transmitter. The BER only reaches $40 \%$ at distances of more than $3 \mathrm{~m}$. Third, increasing the distance between the antennas of the attacker further improves performance-with attacker's antennas placed $1 \mathrm{~m}$ apart, the data signal can be recovered with minimal BER at around 2 meter distance. Note that even if the attacker exhibits a high BER, he might learn some information about the transmitted message. Since the messages are not protected by any form of encryption, the attacker might be able to recover the full message even at a high BER.

So far, we have assumed that the attacker can perfectly align the jamming signal received on both his antennas. We will validate this assumption in our experiments.

\section{G. Summary of our Simulation Results}

Our results show that our attack indeed allows the attacker to recover the data signal in the presence of friendly jamming. While the range of the attack for the $403 \mathrm{MHz}$ case is limited to at most a few meters (depending on the antenna setup of the attacker), the efficiency of the attack increases for higher carrier frequencies such as $2.4 \mathrm{GHz}$ (e.g. IEEE 802.11) if $\overline{D J}$ is kept constant. For these frequencies, the attacker should be able to mitigate the jamming signal for distances of up to $10 \mathrm{~m}$ with a $1 \mathrm{~m}$ spacing between his antennas.

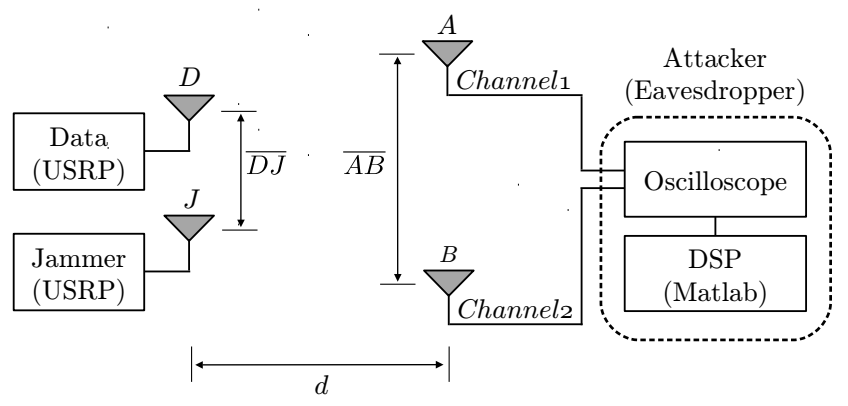

Figure 10. Experimental setup showing the two attacker antennas, the data transmitting USRP and the friendly jammer.

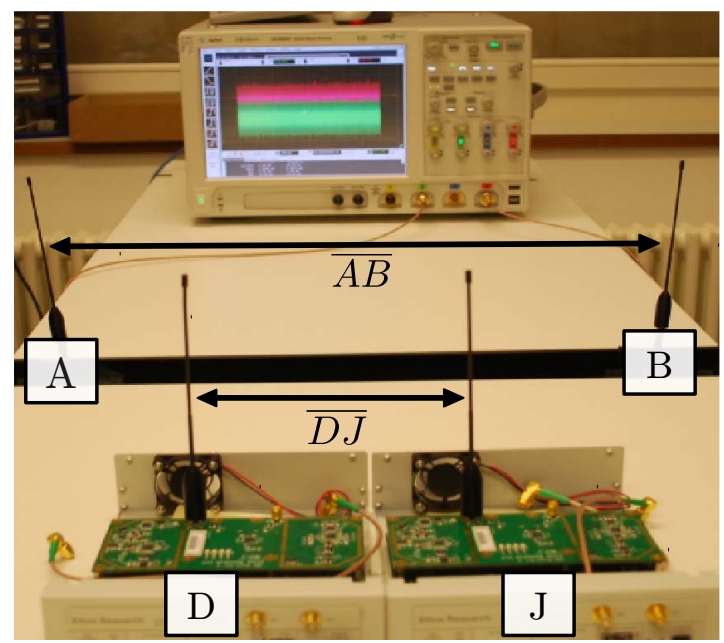

Figure 11. The attacker uses two antennas $(A$ and $B)$ and an oscilloscope in order to record the combined data $(D)$ and jamming $(J)$ signals. For each measurement, the attacker antennas were manually moved around (but maintaining the distance 'd' between $A B$ and $D J$ ) until the data and jamming signal were reasonably separable during post-processing (details in Section V-A).

While these distances might not seem threatening at first, we note that e.g., in the case of medical implants, the communication range of IMD devices is also very limited in normal operations. Our attack shows that friendly jamming fails to protect the confidentiality of communication in the MICS band.

While we concentrated on a specific geometric setup in our evaluation, the attacker is not restricted to the isosceles trapezoid setting (we discuss this more in Section V-A). In addition, the attacker can use more than two antennas to improve his performance.

\section{EXPERIMENTAL ANALYSIS}

In this section, we experimentally verify the attack described in Section III. First, we describe the experimental setup used to demonstrate the feasibility for an attacker to successfully demodulate data (within acceptable bit error rates) in the presence of a friendly jammer. Then we give 


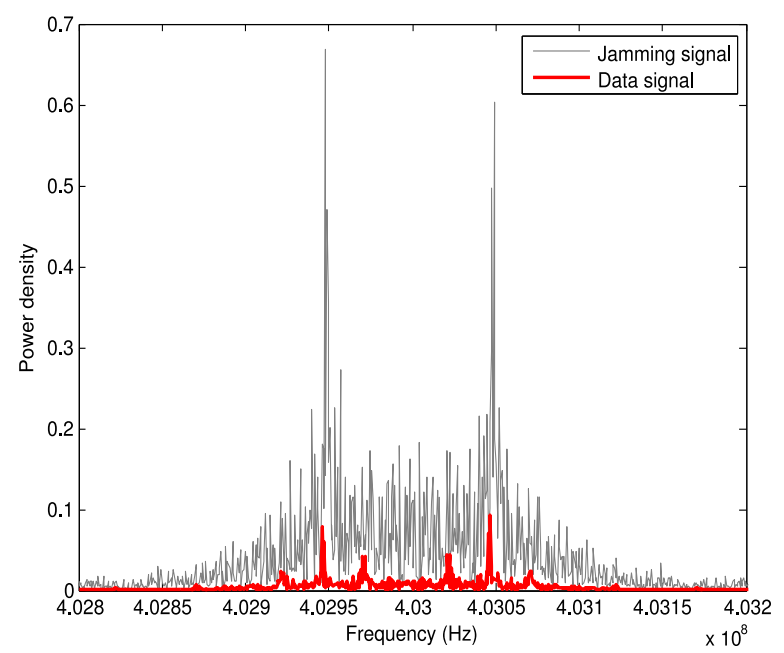

Figure 12. Frequency spectrum of the jammer and data signal.

a brief overview of the evaluation metric used in the experiments. Finally, we present our experimental results and discuss their implications.

\section{A. Experimental Setup}

Our experimental setup consists of an attacker (here, a passive eavesdropper), a data transmitter and a friendly jammer. Figure 10 shows a block schematic of the complete setup and Table II summarizes the system parameters used in the experiments. We further describe the details of our setup below.

1) Data Source and Friendly Jammer: Two Universal Software Radio Peripherals (USRPs) are used to emulate the data transmitter $(D)$ and the friendly jammer $(J)$. Both entities $D$ and $J$ transmit using $0 \mathrm{~dB}$ gain omni directional antennas. A picture of the data-jammer setup is shown in Figure 11. Each data packet is 67 bits long with a 29 bit preamble. The preamble is chosen such that it has high auto-correlation properties. Data is modulated using binary frequency shift keying, i.e., 1's and 0's are transmitted using two discrete frequencies. In our setup, the two frequencies are separated by $100 \mathrm{KHz}$ and upmixed to a RF carrier frequency of $403 \mathrm{MHz}$. The jamming signal is generated by modulating random bits, which results in a spectrum closely matching the spectrum of the data signal (Figure 12). It is ensured that the jamming signal is always transmitted with at least $20 \mathrm{~dB}$ more power with respect to the data signal.

2) Attacker: The attacker is implemented using two omni directional vertical antennas connected to an oscilloscope (Figure 11). For the experiments, the two attacker antennas were placed $\overline{A B}=\{35,50\} \mathrm{cm}$ apart. In the experimental setup, the attacker experiences multipath effects due to reflection in the lab. Thus, the isosceles trapezoid formation used in the LOS simulations does not necessarily provide the optimal results. Consequentially, we allowed the attacker to adapt the antenna placement to maximize performance, while preserving the distance $d$ to $D, J$ and the distance between the attacker antennas $\overline{A B}$. As we will show later, at some locations we were not able to find ideal placements for the antennas of the attacker.

The received signals were recorded using two different oscilloscope channels at a sampling rate of $10 \mathrm{GSa} / \mathrm{s}$ and later processed using Matlab [25].

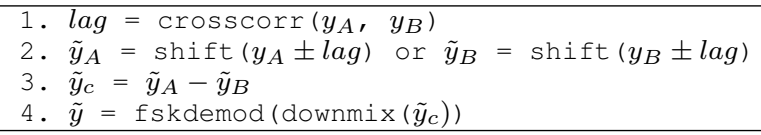

Table I

PSEUDO CODE OF THE JAMMING MITIGATION TECHNIQUE IMPLEMENTED IN MATLAB.

3) Matlab Implementation: First, the signals are bandpass-filtered to exclude unwanted channel activity. Then the power levels of the signals are normalized to match the amplitudes. As the signals are dominated by the jamming components, this improves the match of the two jamming components. The signals are then phase-aligned by cross-correlating both the recorded signals and determining the time lag between the two. Based on the lag, one of the two received signals is appropriately shifted. Since the jamming signal is assumed to have considerably higher power compared to the data signal, the cross-correlation effectively aligns the jamming signal components while keeping the existing phase difference between the data signal components intact. Computing the arithmetic difference between the two aligned signals results in the recovered data signal — this recovered signal is an attenuated or amplified copy of the original data signal as described in Section III. Figure 13 shows the signals received at the two attacker antennas and the recovered data signal post alignment and subtraction. The transmitted data is determined after appropriate synchronization and FSK demodulation of the recovered data signal. If $y_{A}$ and $y_{B}$ are the signals received at the attacker antennas $A$ and $B$, the stepwise Matlab implementation is summarized as pseudo code in Table I.

\section{B. Evaluation Metric}

The attacker's ability to successfully recover the data signal is evaluated based on the bit error rate of the demodulated data from the received signal $\tilde{y}$ when compared to original data in the transmitted signal $s_{d}$. We express the number of bit errors in the recovered data as a percentage of data packet length. In our experiments, we vary the distance between the attacker and $D, J$, and the power levels of the jamming signal relative to data signal. For each configuration, 15 different measurements were recorded and the mean bit error rate computed. 


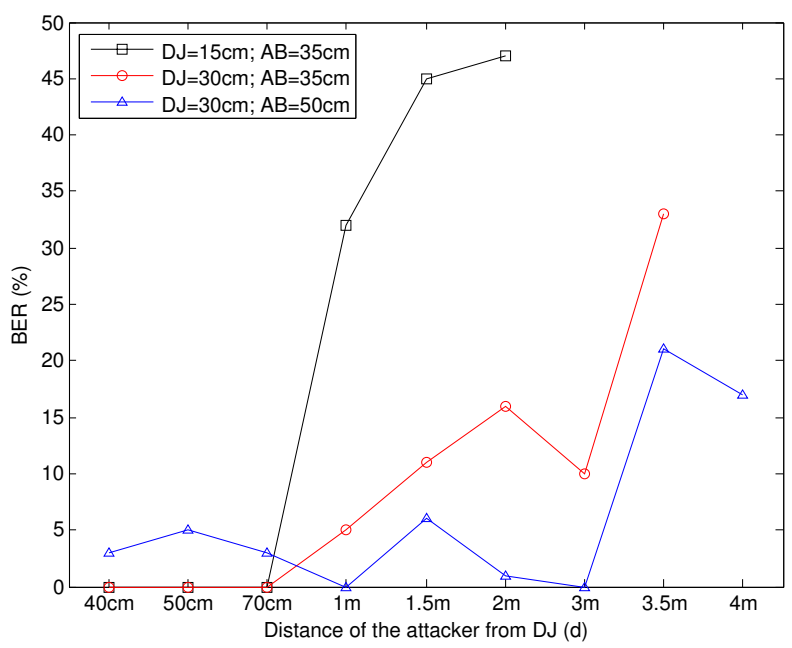

(a)

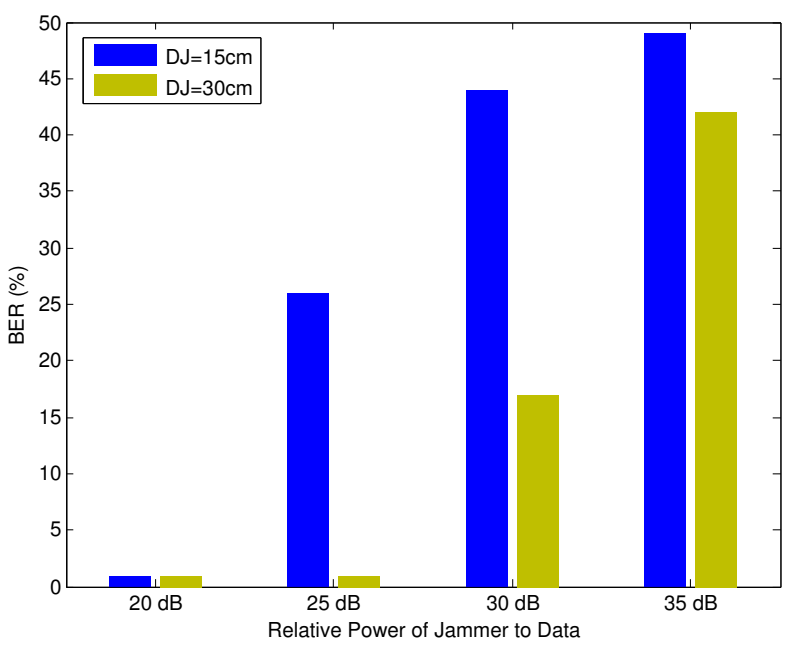

(b)

Figure 14. (a) Bit error rates obtained by the attacker while at various distances away from the data-jammer antenna configuration. The data and the jammer were configured to transmit in the MICS $403 \mathrm{MHz}$ band. The BERs indicated in the plot are arithmetic mean of 20 signal acquisitions at each location. Every data point is an average over 20 measurements and data-jammer transmitting frequencies were centered around $403 \mathrm{MHz}$. (b) Minimum jammer power required to prevent a passive eavesdropper $1 \mathrm{~m}$ away from the data transmitting antenna.

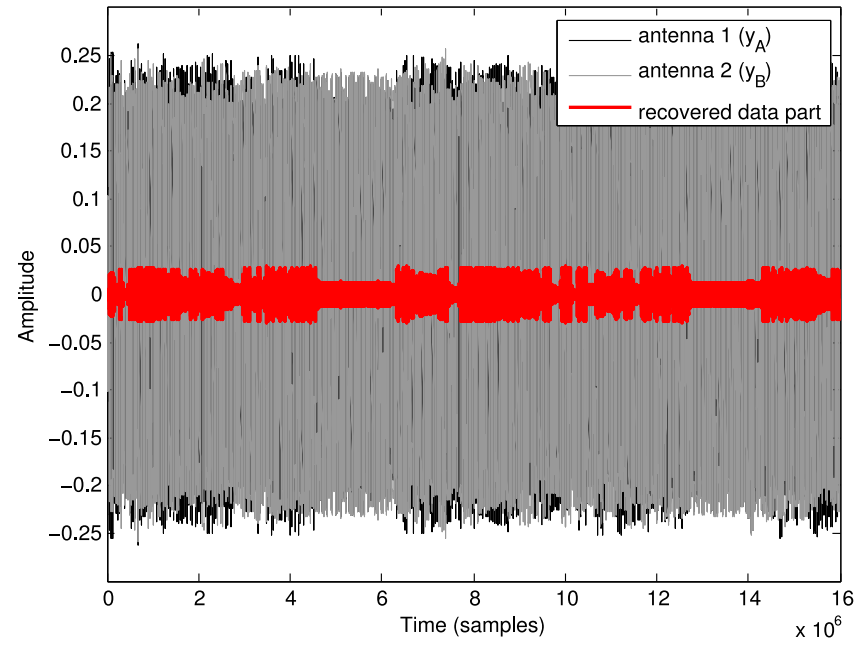

Figure 13. Black and gray waveforms correspond to signals acquired from two receiver antennas. Once the signals were aligned and subtracted, in red we can see the clearly visible, remaining data signal component.

\section{Measurements and Analysis}

In this section, we present our experimental results and briefly discuss their implications.

1) Effect of Attacker's distance: As described in Section III, the relative strength of the recovered data signal degenerates as the distance of the attacker from $D, J$ increases. We experimentally verify our model and determine the maximum distance at which an attacker can successfully recover the data signal in real-world channel settings. The power of the jamming signal is configured to be $20 \mathrm{~dB}$ more than the data signal. For every distance between the

\begin{tabular}{|l|l|}
\hline Parameter & Value \\
\hline Attacker & \\
Antenna type & Omni-directional vertical \\
No. of antennas & 2 \\
Sampling rate & $10 \mathrm{GSa} / \mathrm{s}$ \\
\hline Data transmitter & Omni-directional vertical \\
Antenna type & $403 \mathrm{MHz}$ \\
Carrier frequency & $300 \mathrm{KHz}$ \\
Bandwidth $(\beta)$ & $67 \mathrm{bits}$ \\
Packet length & $150 \mathrm{Kbps}$ \\
Data rate & \\
\hline Jammer & Omni-directional vertical \\
Antenna type & $300 \mathrm{kHz}$ \\
Jamming bandwidth & Spectrum shaped random noise \\
Noise type & $\{20,25,30,35\}$ dB \\
Relative Power of Jammer & \{
\end{tabular}

Table II

SUMMARY OF THE SYSTEM PARAMETERS IN EXPERIMENTAL SETUP.

attacker and $D, J$, two sets of measurements are taken setting the spacing between the data and jammer antennas to $15 \mathrm{~cm}$ and $30 \mathrm{~cm}$. The results of our experiments are shown in Figure 14(a). The results show that the attacker can efficiently recover data for distances greater than one meter by using a small antenna array with $\overline{A B}=30 \mathrm{~cm}$ when the data source and jammer are closer than $\lambda / 2$. By increasing $\overline{A B}$ to $50 \mathrm{~cm}$, we were able to recover data with less than $20 \%$ BER from a distance of $4 \mathrm{~m}$. For smaller values of $\overline{D J}$ or $\overline{A B}$, the BER of the recovered data signal at the attacker increases, as predicted in our simulation results presented in Section III. Due to multipath effects in our lab environment, we were not able to find a good placement for the antennas around $d=1.5 \mathrm{~m}$. 


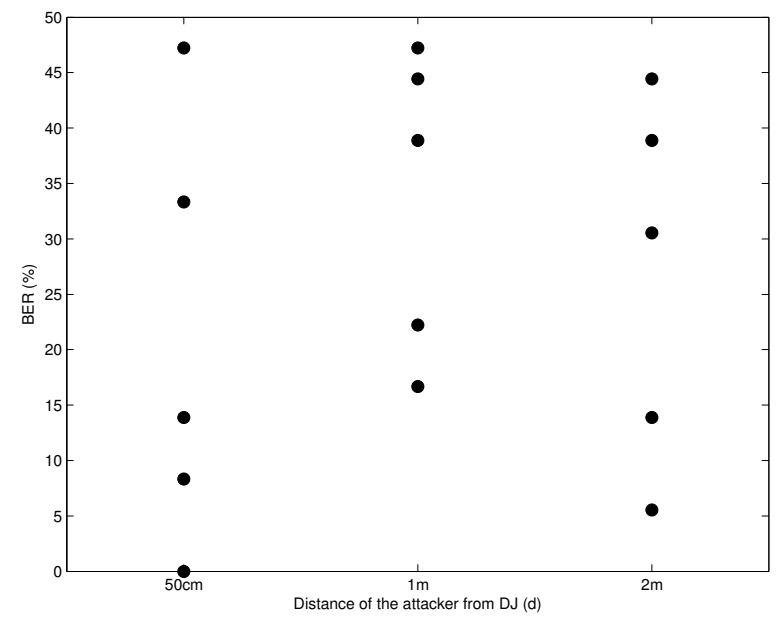

Figure 15. Bit error rates obtained by an attacker for various antenna orientations and positions when the data transmitting antenna $D$ was placed inside a slab of meat to simulate NLOS conditions.

2) Effect of Jamming Signal's Power: In the next set of experiments, we fix the distance between the attacker and $D, J$ to $1 \mathrm{~m}$ and vary the relative power of the jamming signal w.r.t the data signal. The jammer is configured to transmit at $\{20,25,30,35\} \mathrm{dB}$ more than the power of the data signal. Two sets of measurements with the jammer placed at $\overline{D J}=\{15,30\} \mathrm{cm}$ from the data transmitter is taken. For all the measurements, the spacing between the attacker antennas were fixed at $\overline{A B}=50 \mathrm{~cm}$. The results of our experiments is shown in Figure 14(b). We observe that for $\overline{D J}=30 \mathrm{~cm}$, the attacker is able to recover $80 \%$ of the data even with a relative jamming signal power of $30 \mathrm{~dB}$. However, for $\overline{D J}=15 \mathrm{~cm}$, the bit error rate $(\mathrm{BER})$ at the attacker reaches almost $50 \%$.

This shows that the jamming signal is not canceled perfectly by the attacker. We estimate that this effect is due to three possible sources of error: (i) imperfect alignment of the jamming signals before cancellation, (ii) slightly different channels for both receiving antennas in our real world scenario, and (iii) uncorrelated receiving noise at the receivers (the $w$ we ignored earlier). Nevertheless, it can be observed that our attack works in a number of scenarios.

3) For $\overline{D J} \ll \lambda / 2$ : In order to evaluate the attacker's effectiveness in separating the data from the jamming signal when $D$ and $J$ are placed in very close proximity, we experimented with $\overline{D J}=5 \mathrm{~cm}$ (i.e, $\overline{D J}$ at-least an order of magnitude less than $\lambda$ ). We observed BERs close to $50 \%$ at distances greater than $1 \mathrm{~m}$ when using two omnidirectional antennas. However, with directional log-periodic antennas [26] we were able to fully recover the data signal from distances as far as $3 \mathrm{~m}$ from $D J$.

4) In NLOS Conditions: In this set of experiments, we evaluate the ability of an attacker to recover messages in scenarios where there is no LOS between the attacker and the data transmitter. We simulated this setup by sandwiching the data transmitting antenna $(D)$ between $4 \mathrm{~cm}$ of ground beef and a thin $(1 \mathrm{~cm})$ layer of bacon. Since there is no restriction on the type of antenna an attacker can use, we used directional log-periodic antennas for additional gain and to minimize multipath channel effects. The attacker antennas were placed at distances $\{0.5,1,2\} \mathrm{m}$ away from $D$ and $J$. The jamming signal was ensured to be at-least $20 \mathrm{~dB}$ more than the data signal. While our experimental setup resembles the setting from [11], we note that there are several differences. In particular, we use an USRP [27] to generate the data signals instead of an IMD. In addition, we use a different process to generate the spectrum-shaped jamming signal.

Figure 15 shows the BER observed at the attacker for various orientations of attacker antennas $A$ and $B$ keeping his distance $d$ a fixed value. Even though the BER varies for different orientations (which is expected due to non-static channel conditions), the attacker was still able to recover $90 \%$ of the data in certain locations. This shows that there are indeed constellations in space where the confidentiality of data is breached under NLOS conditions. An exhaustive analysis of effects of NLOS multipath channels on friendly jamming schemes is left for future work.

\section{Discussion}

In this section, we reflect on the results of the previous sections and discuss possible extensions and related topics. We start with the impact of medium BER attacks, discuss the impact of multiple friendly jammers, possible countermeasures, effects of SNR and multipath, and future work.

\section{A. Placement of the Antennas}

So far, we have not discussed how the attacker should place his antennas to perform the attack. While finding positions for $A$ and $B$ such that $\delta=\pi$ is easy in the LOS setting without multipath, in real-world scenarios this would require exact knowledge of channel conditions. Nevertheless, we note that we were able to find good enough placements for the antennas in our experiments relatively easily, as the attacker can often still demodulate the data signal with $\delta<\pi / 2$ and less. Our setting differs from traditional benign communication scenarios in the sense that it suffices for the attack to be successful some times, not necessarily all the time. Also, unlike communication partners in benign communication, we assume that the attacker can afford to adapt the antenna placement for changing channel conditions. This allows us to operate in more favorable settings than the average fading channel. We also note that the process of placing the antennas could be automated with a mechanized antenna array, similar to [28].

\section{B. Partial Information Leakage}

In our simulation model and experiments we used BER as an evaluation metric. While the consequences of a very low 
BER (the attacker completely breaks confidentiality) and a BER of 0.5 (confidentiality remains intact) at the attacker are clear, we discuss the implications of partial information leakage-for example, a BER of 0.2 at the attacker. In this scenario, the attacker will successfully recover $80 \%$ of the bits. In many cases, this leads to the attacker completely breaking confidentiality, e.g., a patient's name. In addition, if the IMD sends the same data again (e.g. in a repeated message), the attacker will be able to use this additional information to recover the patient's name, thus completely breaking the system's confidentiality.

\section{Multiple Friendly Jammers}

In this paper, we discussed friendly jamming schemes that rely on a single jammer. This is a commonly used system model when the goal is confidentiality, as the legitimate receiver should be able to demodulate the data. In most cases, the legitimate receiver also acts as the friendly jammer. Schemes that use multiple jammers do not achieve confidentiality of legitimate messages but are only able to restrict unauthorized entities from using the communication medium. To the best of our knowledge, the only scheme which relies on multiple jammers for confidentiality is one where the jammers form a "jamming fence" around a protected area [15]. Nevertheless, in these settings, multiple jammers are used to cover a greater area and not to improve system's confidentiality. Thus, these schemes assume that a single jammer is enough to protect the confidentiality of the messages. As such, we follow that our attack could also be used to attack these proposals - at least for positions which are only covered by a single jammer.

\section{Countermeasures}

From our analysis and experiments, we can draw several conclusions on possible countermeasures against our attack. While these do not guarantee complete confidentiality of the system, they decrease the attacker's probability of success. In particular, our simulations and experimental results underline the impact of close placement of $J$ to $D$. As we have shown, simply choosing $D J<\lambda / 2$ does not prevent the attacker from receiving the data. Nevertheless, the BER of the attacker rises with decreasing $\overline{D J}$. Therefore, $\overline{D J}$ should always be chosen to be as small as possible. As $\lambda$ depends on the carrier frequency, $D J$ must be be smaller for higher frequencies to provide the same level of protection. Therefore, sending data signals in the MICS bands is easier to protect than, for example, IEEE 802.11 signals. In addition, we estimate that multiple jammers will at least force the attacker to use more antennas for his receiving array. As discussed before, the problem here is to ensure that the intended receiver can still receive the message despite the presence of multiple friendly jammers.

\section{E. SNR and Multipath Effects}

In our simulations while computing BER, we assume a certain SNR at the attacker in the absence of the jammer. This is because a performance evaluation of our attack only makes sense if the attacker can receive the message in the absence of a friendly jammer. For our simulations, our estimations of the SNR at the attacker was $16 \mathrm{~dB}$. The attacker can improve his SNR by using better receiving equipment (e.g. low noise amplifiers, better ADC, high-gain antennas etc.). This would even improve the efficiency of our attacks.

Another important factor affecting the performance of our attacks are the multipath effects. In our analysis, we concentrate on modeling line-of-sight channels. In practice, multipath effects can degrade the performance of our attack by introducing additional phase shifts or signal fading effects. Nevertheless, our experiments, conducted in realworld settings under the influence of multipath effects, still yield realistic attacker scenarios. However, the performance for non line-of-sight scenarios needs to be further explored. It is to be expected that in the worst case, the attacker still has a probabilistic chance of randomly being at a correct position for his attack (similar to malicious interference attacks in [29]).

\section{F. Effect of Signal Bandwidth and Modulation Scheme}

We noted earlier that higher signal bandwidth results in a more distorted reconstructed data signal.

To understand the effects of signal bandwidth on our proposed attack, we consider the ratio $r=\beta / f_{c}$ of data signal bandwidth $\beta$ and its carrier frequency $f_{c}$. While for MICS signals this ratio is around 0.00075, in UWB communications $r$ could approach 0.25 . The ratio directly relates to the length of a baseband symbol, expressed in carrier periods. In our presented cancellation scheme, the two data components are subtracted with some phase shift, ideally $\lambda / 2$. For signals with very low bandwidth, adding a $\lambda / 2$-shifted version of itself completely cancels the signal and subtracting it almost doubles the amplitude. For signals with higher bandwidth, subtracting a $\lambda / 2$-shifted version will not completely cancel the signal, since the amplitudes of the carrier will change considerably with each period.

Intuitively, with lower signal bandwidth, the signal is more similar to its $\lambda / 2$-shifted version, and thus the cancellation (or amplification) will be better. Figure 16 shows how different values of $r$ affect the cancellation rate.

In this work we focused on low-bandwidth FSK as modulation scheme. We leave the evaluation of other modulations schemes (e.g. high bandwidth UWB signals) for future work.

\section{G. Jamming for Access Control}

Throughout the paper we focused on jamming for confidentiality. Jamming can also be used for achieving access control, i.e., using friendly jamming in order to deny access 


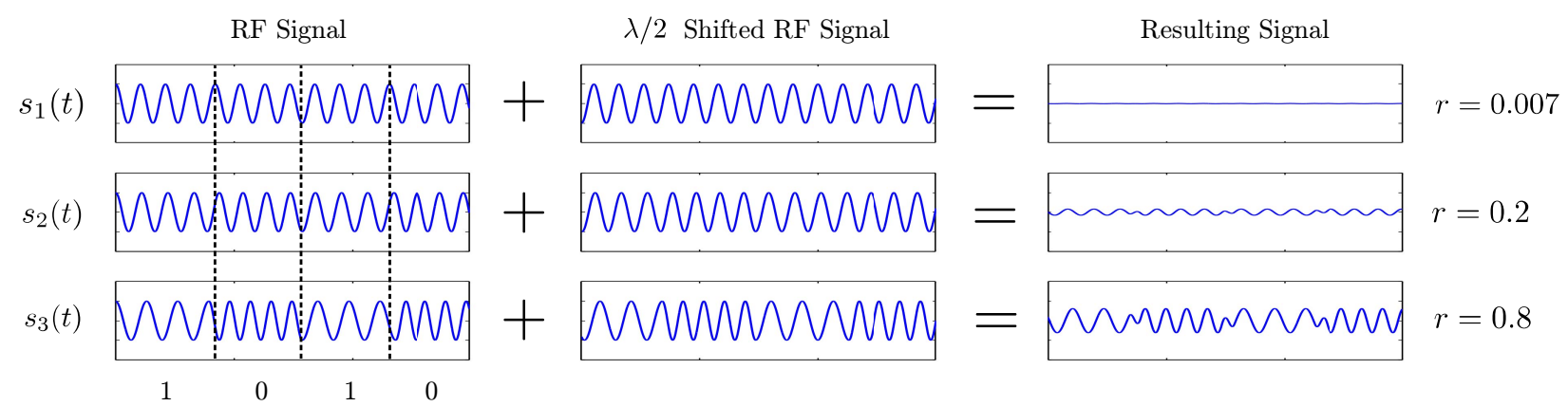

Figure 16. Effect of the ratio between bandwidth and carrier frequency $r=\beta / f_{c}$ on cancellation effectiveness. Cancellation effect is larger for smaller values of $r$.

to a protected device. Existing schemes implement one of the following jammer classes.

- Fast jammer. A friendly jammer demodulates signals intended for the protected device in real-time. Upon demodulating and inspecting parts of the frame, a choice is made whether or not the frame is allowed. In case the frame does not conform to a pre-defined access control policy, the remaining portion of the frame will be jammed. Such a strategy prevents the protected device from successfully demodulating a complete adversarial query.

- Slow jammer. The friendly jammer listens for messages destined for the protected device but does not start jamming in mid-frame. The jammer receives the whole frame and, in case the access control policy is violated, starts jamming the reply of the protected device.

$\mathrm{Xu}$ et al. propose a scheme for securing IMD devices against access from unauthorized sources [12]. Security is enforced by a specialized device which employs friendly jamming - the Guardian. The authors argue it is more reliable and effective to let the Guardian jam replies by the IMD, instead of directly jamming query messages issued by the attacker. The Guardian device can effectively set it's own jamming parameters because it knows the transmission power and reply timing information of the protected IMD.

Rieback et al. proposed the concept of selective RFID jamming to enforce access control of RFID tags [8], [9]. An external friendly jammer listens for queries towards protected RFID tags. If the query is not allowed, based on an access control policy, the tag reply is jammed.

The above works jam replies made by the protected device, but not adversarial queries - they implement the slow jammer approach.

Martinovic et al. proposed a scheme based on a fast jammer. The authors use the concept of friendly jamming in sensor networks to enforce access control by jamming unauthenticated frames in mid-air [10]. The proposed scheme prevents protected devices from successfully demodulating unauthorized queries, i.e., it implements the fast jammer approach.

Our proposed attack can be used against access control schemes which implement the slow jammer approach. An adversary can mount an active attack by sending unsanctioned queries to protected devices. Friendly jammers detect such queries and jam device replies. However, our scheme is then used to remove the jamming component from the reply. By doing so, an adversary is able to bypass the access control policy and communicate with the protected device in the same way as if a friendly jammer was not present.

\section{CONCLUSION}

In this work, we revisit the confidentiality guarantees provided by friendly jamming schemes. We focused on the assumption that the attacker cannot recover the confidential message if the friendly jammer and the message source are in close proximity. We showed, both analytically and experimentally, that this assumption does not hold. In our experiments, performed in the MICS band, we demonstrated the successful recovery of confidential messages from up to $3 \mathrm{~m}$ away, when a friendly jammer and the source were placed only few centimeters apart. Our results show that friendly jamming schemes have some fundamental security limitations when they are used to achieve confidentiality.

\section{ACKNOWLEDGMENTS}

This work was funded by the Swiss National Science Foundation (SNSF) under the grant 200020_129605.

\section{REFERENCES}

[1] R. Negi and S. Goel, "Secret communication using artificial noise," in IEEE Vehicular Technology Conference, 2005.

[2] S. Goel and R. Negi, "Guaranteeing secrecy using artificial noise," IEEE Transactions on Wireless Communications, vol. 7, no. 6, pp. 2180-2189, 2008.

[3] A. Araujo, J. Blesa, E. Romero, and O. Nieto-Taladriz, "Cooperative jam technique to increase physical-layer security in CWSN," in COCORA 2012, The Second International Conference on Advances in Cognitive Radio, 2012, pp. 11-14. 
[4] L. Dong, Z. Han, A. Petropulu, and H. Poor, "Cooperative jamming for wireless physical layer security," in Proceeding of IEEE Workshop on Statistical Signal Processing (SSP), sept 2009, pp. 417-420.

[5] X. Tang, R. Liu, P. Spasojevic and, and H. Poor, "Interference assisted secret communication," IEEE Transactions on Information Theory, vol. 57, no. 5, pp. 3153 -3167, May 2011.

[6] J. Vilela, M. Bloch, J. Barros, and S. McLaughlin, "Friendly jamming for wireless secrecy," in Proceedings of the IEEE International Conference on Communications (ICC). IEEE, 2010, pp. 1-6.

[7] —- "Wireless secrecy regions with friendly jamming," Information Forensics and Security, IEEE Transactions on vol. 6, no. 2, pp. $256-266$, june 2011.

[8] M. R. Rieback, B. Crispo, and A. S. Tanenbaum, "Keep on blockin' in the free world: Personal access control for lowcost RFID tags," in Proc. 13th International Workshop on Security Protocols. LNCS, Apr 2005.

[9] — - "RFID guardian: A battery-powered mobile device for RFID privacy management," in Proc. 10th Australasian Conf. on Information Security and Privacy (ACISP 2005), ser. LNCS, vol. 3574. Springer-Verlag, July 2005, pp. 184-194.

[10] I. Martinovic, P. Pichota, and J. Schmitt, "Jamming for good: A fresh approach to authentic communication in wsns," in Proceedings of the second ACM conference on Wireless network security. ACM, 2009, pp. 161-168.

[11] S. Gollakota, H. Hassanieh, B. Ransford, D. Katabi, and $\mathrm{K}$. Fu, "They can hear your heartbeats: Non-invasive security for implanted medical devices," in Proceedings of the ACM SIGCOMM Conference, August 2011.

[12] F. Xu, Z. Qin, C. Tan, B. Wang, and Q. Li, "Imdguard: Securing implantable medical devices with the external wearable guardian," in INFOCOM, 2011 Proceedings IEEE. IEEE 2011, pp. 1862-1870.

[13] M. Wilhelm, I. Martinovic, J. B. Schmitt, and V. Lenders, "WiFire: a firewall for wireless networks," in Proceedings of the ACM SIGCOMM Conference, 2011, pp. 456-457.

[14] C. Kuo, M. Luk, R. Negi, and A. Perrig, "Message-in-a-bottle: user-friendly and secure key deployment for sensor nodes," in Proceedings of the 5th international conference on Embedded networked sensor systems. ACM, 2007, pp. 233-246.

[15] S. Sankararaman, K. Abu-Affash, A. Efrat, S. D. ErikssonBique, V. Polishchuk, S. Ramasubramanian, and M. Segal, "Optimization schemes for protective jamming," in Proceedings of the ACM International Symposium on Mobile Ad Hoc Networking and Computing (MobiHoc), 2012, pp. 65-74.
[16] H. Al-Hassanieh, "Encryption on the air: non-invasive security for implantable medical devices," Ph.D. dissertation, Citeseer, 2011.

[17] A. Molisch, Wireless Communications. Wiley, 2011.

[18] S. Leung-Yan-Cheong and M. Hellman, "The gaussian wiretap channel," Information Theory, IEEE Transactions on, vol. 24, no. 4, pp. 451-456, 1978.

[19] X. Zhou and M. McKay, "Physical layer security with artificial noise: Secrecy capacity and optimal power allocation," in International Conference on Signal Processing and Communication Systems (ICSPCS). IEEE, 2009, pp. 1-5.

[20] O. Koyluoglu and H. El Gamal, "On the secrecy rate region for the interference channel," in Proceedings of the International Symposium on Personal, Indoor and Mobile Radio Communications (PIMRC). IEEE, 2008, pp. 1-5.

[21] S. Goel and R. Negi, "Secret communication in presence of colluding eavesdroppers," in Proceedings of the IEEE Military Communications Conference (MILCOM), 2005, pp. 1-6.

[22] P. Pinto, J. Barros, and M. Win, "Wireless physical-layer security: The case of colluding eavesdroppers," in Proceedings of the IEEE International Symposium on Information Theory (ISIT), 2009, pp. 2442-2446.

[23] W. Jakes, Microwave mobile communications, ser. IEEE Press classic reissue. IEEE Press, 1974.

[24] D. Tse and P. Viswanath, Fundamentals of wireless communication. New York, NY, USA: Cambridge University Press, 2005.

[25] "Matlab - a numerical computing environment," The MathWorks, Inc; www.mathworks.com.

[26] Ettus, "Log periodic pcb directional antenna," https://www.ettus.com/product/details/LP0410.

[27] - "Universal software radio peripheral (USRP)," http://www.ettus.com.

[28] T. Vo-Huu, E.-O. Blass, and G. Noubir, "Counter-jamming using mixed mechanical and software interference cancellation," in Proceedings of the ACM conference on Security and Privacy in Wireless and Mobile Networks, 2013.

[29] C. Pöpper, N. O. Tippenhauer, B. Danev, and S. Čapkun, "Investigation of signal and message manipulations on the wireless channel," in Proceedings of the European Symposium on Research in Computer Security, 2011. 